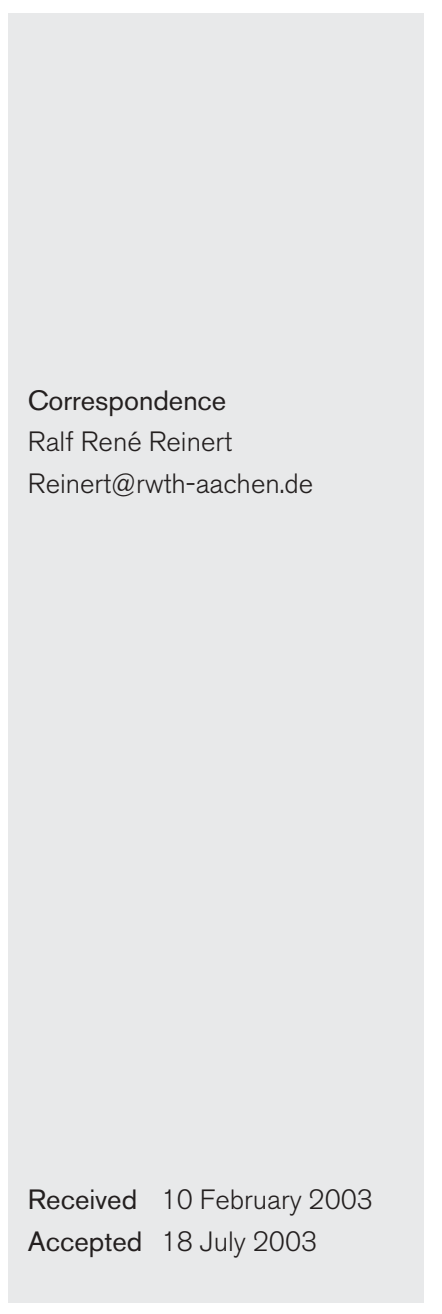

\section{Characterization of German penicillin non-susceptible serotype 23F pneumococci using multilocus sequence typing}

\author{
Ralf René Reinert, ${ }^{1}$ Stefanie Muckel, ${ }^{1}$ Adnan Al-Lahham, ${ }^{1}$ Brian G. Spratt, ${ }^{2}$ \\ Angela B. Brueggemann, ${ }^{3}$ Regine Hakenbeck ${ }^{4}$ and Rudolf Lütticken ${ }^{1}$ \\ ${ }^{1}$ Institute of Medical Microbiology, National Reference Center for Streptococci, University Hospital, \\ D-52057 Aachen, Germany \\ 2 Department of Infectious Disease Epidemiology, Imperial College School of Medicine, St Mary's \\ Campus, London W2 1PG, UK \\ ${ }^{3}$ Department of Microbiology, University of Oxford, and Department of Public Health \& Primary Care, \\ John Radcliffe Hospital, Headington, Oxford OX3 9DU, UK \\ ${ }^{4}$ Institute of Microbiology, University of Kaiserslautern, Germany
}

\section{INTRODUCTION}

Penicillin non-susceptible Streptococcus pneumoniae strains were first reported from Germany in 1992 (Reinert et al., 1992). Within the last 5 years, an increase in both penicillin and macrolide resistance has been observed among $S$. pneumoniae in Germany, as documented by three surveillance studies performed by the National Reference Center for Streptococci. In a nationwide study of invasive pneumococcal disease in adults (study B), the proportion of strains with reduced susceptibility to penicillin (MIC $\geqslant 0.12 \mu \mathrm{g} \mathrm{ml}^{-1}$ ) increased from $1.8 \%$ in 1992 to $5.8 \%$ in 2000 (Reinert et al., 2002). In an ongoing population-based nationwide surveillance study of invasive disease in children (study A), which started in 1997 and covered all paediatric hospitals and clinical microbiology laboratories in Germany, ten strains ( $3.3 \%)$ were found to be penicillin-intermediate (MIC $0 \cdot 12-$ $1.0 \mu \mathrm{g} \mathrm{ml}^{-1}$ ), but no penicillin-resistant strains (MIC $>2 \mu \mathrm{g} \mathrm{ml}^{-1}$ ) were detected (von Kries et al., 2000). During

Abbreviations: MLEE, multilocus enzyme electrophoresis; MLST, multilocus sequence typing; NCCLS, National Committee for Clinical Laboratory Standards; SLV, single locus variant; ST, sequence type. the same period, outpatient pneumococcal isolates were collected prospectively by 19 clinical microbiology laboratories in Germany between November 1998 and April 1999 (study C), with $6 \cdot 8 \%(n=65)$ of 961 pneumococcal strains showing reduced susceptibility to penicillin (Reinert et al., 2001).

Multilocus sequence typing (MLST) is a recently developed technique that produces unambiguous molecular typing data that can be transmitted electronically via the Internet (Enright \& Spratt, 1998; Maiden et al., 1998). Using this procedure, nucleotide sequences of $\sim 450 \mathrm{bp}$ internal fragments of seven housekeeping genes are determined for each isolate. Different sequences at each locus are assigned as distinct alleles and, for each isolate, alleles at the seven loci define the allelic profile or sequence type (ST). The method is highly portable, as any laboratory can compare sequences at the seven loci in their isolates with those in a central database on the World Wide Web (http://www.mlst.net) and obtain the allelic profile of each isolate. The present study uses this technique to analyse clinical strains of penicillin nonsusceptible, serotype 23F S. pneumoniae isolated in Germany between 1992 and 2000. 


\section{METHODS}

Strains. Forty-five single patient isolates of serotype $23 \mathrm{~F}$ pneumococci that were penicillin-intermediate or -resistant were collected between 1992 and 2000 from three national surveillance projects in Germany (Table 1). Twelve isolates were recovered from blood, three from cerebrospinal fluid, 17 from respiratory specimens and 13 from other or unknown sources. In addition, we scanned the MLST database, which includes the allelic profiles of over 700 pneumococci, for pneumococcal serotype 23F infections (Enright \& Spratt, 1998).

Susceptibility testing and serotyping. Susceptibility testing was performed by using the broth microdilution method as recommended by the National Committee for Clinical Laboratory Standards (NCCLS, 2000). Commercially manufactured plates (Micronaut-S; Merlin Diagnostics) that contained penicillin, cefotaxime, erythromycin, clindamycin, tetracycline and cation-adjusted Mueller-Hinton broth (Oxoid) plus $5 \%$ lysed horse blood (Oxoid) were utilized. MICs were determined following incubation at $35^{\circ} \mathrm{C}$ for $20-24 \mathrm{~h}$ in ambient air. $S$. pneumoniae ATCC 49619 was used as a control strain. Isolates were stored at $-70{ }^{\circ} \mathrm{C}$ on porous beads (Microbank; Mast Diagnostics). Susceptibility to cotrimoxazole was determined by means of E test strips (Viva Diagnostica). Current NCCLS interpretive criteria were used to define antimicrobial resistance (NCCLS, 2000).

Pneumococcal strains were serotyped by Neufeld's Quellung reaction (Neufeld, 1902), using type and factor sera provided by the Statens Serum Institut, Copenhagen, Denmark. The serotype distribution of this collection of penicillin non-susceptible pneumococci was analysed with reference to all pneumococci collected in three German multicentre studies (von Kries et al., 2000; Reinert et al., 2001, 2002).

DNA purification, PCR protocols and MLST. PCR amplification was carried out on chromosomal DNA by using an extension time of $30 \mathrm{~s}$ and an annealing temperature of $50^{\circ} \mathrm{C}$ with Taq polymerase (Roche Diagnostics). DNA fragments were purified by using the QIAquick system (Qiagen) and sequencing reactions were carried out in each direction by using the primers that had been used for initial PCR amplification. After gel electrophoretic separation of $8 \mu \mathrm{l} \mathrm{PCR} \mathrm{product}$ in $1 \%$ agarose, amplicons were visualized and their length determined by using UV transillumination. For gel documentation, the BioDocAnalyze system (Biometra) was used.

MLST was carried out as described previously. Briefly, internal fragments of the aroE, $g d h, g k i, r e c \mathrm{P}, s p i, x p t$ and $d d l$ genes were amplified by PCR from chromosomal DNA with the primer pairs described by Enright \& Spratt (1998).

Assignment of alleles at each locus was carried out with software available at the pneumococcal MLST website (http://www.mlst.net). Alleles at each of the seven loci provide the allelic profile of each isolate and also define their ST. Allelic profiles are shown as the alleles at each of the seven loci, in the order aroE, gdh, gki, recP, spi, xpt, ddl. Allelic profiles of the German isolates were compared with each other, as well as with other isolates in the pneumococcal MLST database, by using software available at the MLST website.

Detection of resistance genes. For amplification of tet $M$, primers originally published by Provvedi et al. (1996) with the sequences 5'TGGAATTGATTTATCAACGG-3' (positions 2496-2515) and 5'TTCCAACCATACAATCCTTG-3' (positions 3575-3556) were used. For amplification of tet $O$, the primers $5^{\prime}$-GGCACAGACCCGTA TACTGTT-3' (positions 442-462) and 5'-TTAAAAGAGGGTCGC CATCTG-3' (positions 1230-1250) were used (Widdowson et al., 1996; Widdowson \& Klugman, 1998). For detection of erm and mef, primers described by Trieu-Cuot et al. (1990) and by Tait-Kamradt et al. (1997) with the sequences (erm) 5' -CGAGTGAAAAAGTACTCAACC3' (positions 362-382) and 5'-GGCGTGTTTCATTGCTTGATG-3'

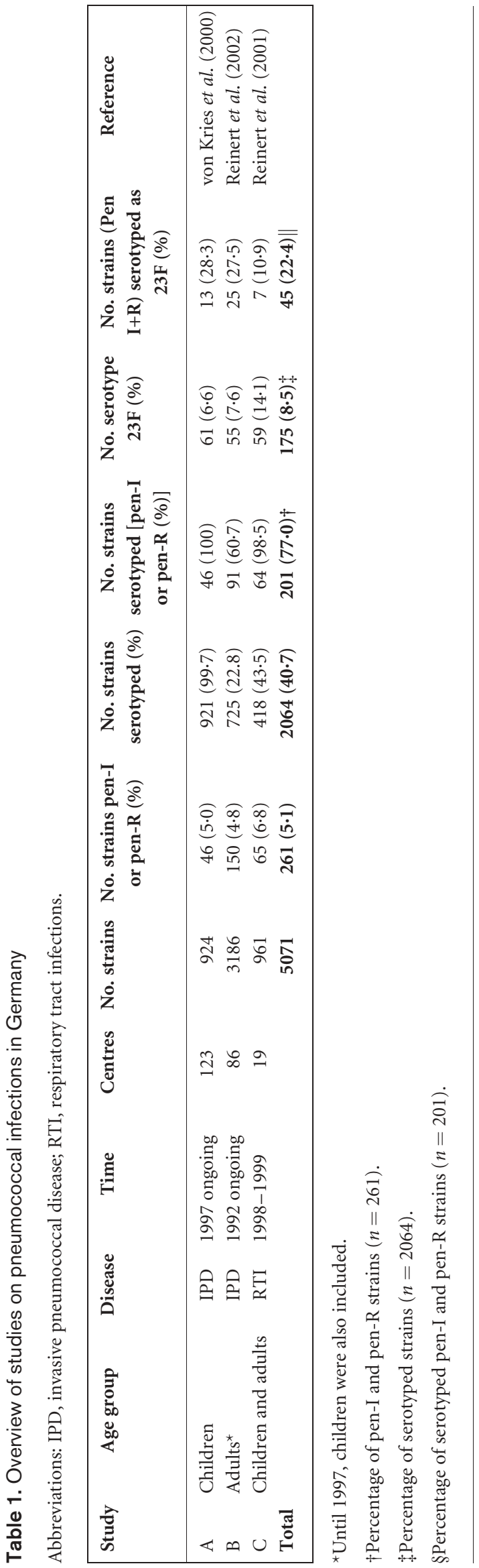


(positions 978-958) and (mef) 5' -AGTATCATTAATCACTAGTGC-3' (positions 57-77) and 5'-GTAATAGATGCAATCACAGC-3' (positions 551-532) were selected (Reinert et al., 2001).

\section{RESULTS AND DISCUSSION}

Serotyping data were available for 201 representative penicillin non-susceptible strains of pneumococci collected in three German multicentre studies (Table 1) (von Kries et al., 2000; Reinert et al., 2001, 2002). Serotype 23F was the predominant serotype $(n=45,22 \cdot 4 \%)$, followed by $6 \mathrm{~B}$ $(n=30,14.9 \%), 9 \mathrm{~V}(n=19,9.5 \%), 19 \mathrm{~A}(n=18,9 \%)$, $19 \mathrm{~F}(n=17,8.5 \%), 14(n=14,7 \%), 6 \mathrm{~A}(n=9,4.5 \%), 15 \mathrm{~B}$ $(n=6,3 \%)$ and others $(n=43,21 \cdot 4 \%)$.

Table 2 shows demographic, antimicrobial susceptibility and MLST data for individual strains. The following sequence types were found: ST $81(n=21,46 \cdot 2 \%)$, ST $277(n=8$, $17 \cdot 8 \%)$, ST $242(n=2)$, ST $142(n=2)$, ST $323(n=1)$ and novel STs $(n=11,24 \cdot 4 \%)$.

ST 81 strains were isolated in Germany each year from 1992 to 1999. ST 81 (the ST of the Spanish ${ }^{23 \mathrm{~F}}$-1 clone; McGee et al., 2001) appears to be geographically distributed throughout Germany and was recovered from both invasive and noninvasive disease sources. Seven strains among the group of German ST 81 strains were high-level penicillin-resistant $\left(\mathrm{MIC} \geqslant 2 \mu \mathrm{g} \mathrm{ml}^{-1}\right)$.

Most strains $(n=15,71.4 \%)$ of ST 81 were susceptible to erythromycin (MIC $\leqslant 0 \cdot 25 \mu \mathrm{g} \mathrm{ml}^{-1}$ ), but $90 \%$ of isolates (19 of 21 ) were resistant to tetracycline (MIC $\geqslant 4 \mu \mathrm{g} \mathrm{ml}^{-1}$, all tet $M$-positive). All German isolates of the Spanish ${ }^{23 \mathrm{~F}}-1$ clone had the typical allelic profile of this clone (ST 81).

The second most prevalent penicillin-resistant 23F clone in Germany is ST 277. Eight strains (17.8\%) had ST 277 and another four were very closely related, differing from the allelic profile of this ST at only a single locus [single locus variants (SLVs)]. Only one isolate of ST 277 can be found in the MLST database; this is a penicillin-resistant serogroup 23 strain isolated in the Netherlands (strain JJ279-23). Strains of ST 277 were mostly isolated (four of eight ST 277 strains) in the extreme west of Germany, whilst the other strains were detected in central and southern Germany. No SLVs of ST 81 were detected, whereas four SLVs of ST 277 (STs 359 and 360, one each; 361, two strains) were found to be present in Germany. All SLVs showed alterations in the xpt gene, harbouring allele 6 (strains PS 275 and PS 278; ST 361), allele 20 (RKI 425; ST 359) or allele 65 (RKI 682; ST 360). All strains of ST 277 and its SLVs were intermediately penicillinresistant and were erythromycin- and tetracycline-susceptible.

Two strains (PS 1953 and PS 2006) were identified as ST 242; one further strain was an SLV of this ST (RESP 95; ST 362). Interestingly, ST 242 has only been reported from Taiwan to date and is now designated the Taiwan ${ }^{23 \mathrm{~F}}-15$ clone (Shi et al., 1998). The two ST 242 isolates in the German strain collection were intermediately penicillin-resistant and were erythromycin- and tetracycline-resistant, as are Taiwanese isolates of this clone. The ST 242 strains showed the MLS type of resistance (erm-positive), whereas the SLV (ST 362) was mef-positive.

Two strains in the German strain collection (RKI 530 and RKI 531) belong to ST 142, which is defined by a single penicillin-resistant serotype $23 \mathrm{~F}$ strain ( $\mathrm{M}$ 402) that was isolated in 1998 in Spain. ST 142 is a SLV of ST 277, which is widespread in Germany. Interestingly, both ST 142 isolates were isolated in the same city, Dortmund, in 1999. Strains of ST 142 are susceptible to erythromycin and clindamycin; however, in contrast to the Spanish isolate, they are resistant to tetracycline.

One strain (RESP 798; ST 355) is an SLV to the high-level cephalosporin-resistant serotype $23 \mathrm{~F}$ clone from the US (Tennessee $^{23 \mathrm{~F}}-4$; pneumococcal network, accession no. ATCC 51916). In contrast to the Tennessee ${ }^{23 \mathrm{~F}}-4$ clone, the German isolate remained susceptible to cephalosporins. Five of the German strains had allelic profiles that were not related closely (SLVs) to any isolates in the MLST database (STs 353, $354,356,357,358)$.

Penicillin resistance in pneumococci is now an infectious disease problem of international significance. Penicillin resistance rates vary even between neighbouring European countries (Klugman et al., 1997; Pradier et al., 1997). An extremely high level of penicillin resistance is reported from southern and south-eastern Europe, whereas the level of pneumococcal $\beta$-lactam resistance remains low in Germany (Reinert et al., 1995, 2001, 2002; von Kries et al., 2000), the Netherlands (Hermans et al., 1997), the northern European countries (Nielsen \& Henrichsen, 1992; Henriques et al., 2000) and the UK (Johnson, 1998; Andrews et al., 1999).

MLST now offers an excellent tool for unambiguous characterization of pneumococci by sequencing approximately 450 bp fragments of seven housekeeping loci (Enright \& Spratt, 1998). MLST is particularly useful for comparing penicillin- and multiresistant isolates recovered in one geographical region with those described elsewhere (Zhou et al., 2000).

The present study showed serotype $23 \mathrm{~F}$ to be the most predominant penicillin non-susceptible serotype isolated in Germany. Strains from three nationwide surveys were included in the analysis. As study A (population-based study covering all laboratories in Germany) and study B (Table 1) are nationwide studies, the penicillin non-sensitive serotype $23 \mathrm{~F}$ pneumococcal strains included in the present analysis are representative isolates for invasive pneumococcal disease in Germany. In contrast, representativeness of the present analysis for pneumococcal respiratory tract infections may be limited.

Among all serotype $23 \mathrm{~F}$ pneumococci that were recovered in Germany from 1992 to 2000, MLST unambiguously demonstrated that the Spain ${ }^{23 \mathrm{~F}}-1$ clone (ST 81) contributed significantly to the spread of penicillin resistance in Germany. Representatives of ST 277 and SLVs of this ST are also observed in Germany. A few isolates were indistinguishable 
Table 2. Characteristics of 45 penicillin non-susceptible pneumococcal serotype $23 \mathrm{~F}$ strains isolated in Germany in 1992-2000, in comparison to international reference strains Strains from the MLST strain collection are marked with bold face. Abbreviations: F.S., federal state; CLI, clindamycin; CTX, cefotaxime; ERY, erythromycin; PEN, penicillin G; SXT, cotrimoxazole; TET, tetracycline; ST, sequence type; ND, no data; B, Berlin; BA, Bavaria; BW, Baden-Württemberg; HE, Hesse; HH, Hamburg; LS, Lower Saxony; NRW, North Rhine-Westphalia; SA, Saxony; TU, Thuringia; BS, bronchial secretion; conj., conjunctiva; CSF, cerebrospinal fluid; Nasop., nasopharynx; PF, pleural fluid; f, female; m, male.

\begin{tabular}{|c|c|c|c|c|c|c|c|c|c|c|c|c|c|c|c|c|c|c|c|c|}
\hline \multirow[t]{2}{*}{ Strain } & \multirow{2}{*}{$\begin{array}{c}\text { Isolation } \\
\text { year }\end{array}$} & \multirow[t]{2}{*}{ Town } & \multirow[t]{2}{*}{ F.S. } & \multirow[t]{2}{*}{ Source } & \multirow[t]{2}{*}{ Sex } & \multirow[t]{2}{*}{ Age } & \multicolumn{6}{|c|}{ MIC $\left(\mu \mathrm{g} \mathrm{ml}^{-1}\right)$ of: } & \multicolumn{7}{|c|}{ Allelic profile } & \multirow[t]{2}{*}{ ST } \\
\hline & & & & & & & PEN & CTX & ERY & CLI & TET & SXT & aroE & $g d h$ & $g k i$ & recP & spi & $x p t$ & $d d l$ & \\
\hline \multicolumn{21}{|l|}{ ST 81} \\
\hline PS 16 & 1992 & Göttingen & LS & Conj. & $\mathrm{m}$ & 2 & 1 & 1 & $0 \cdot 06$ & $0 \cdot 06$ & 32 & 16 & 4 & 4 & 2 & 4 & 4 & 1 & 1 & 81 \\
\hline PS 62 & 1992 & Munich & BA & BS & $\mathrm{m}$ & 62 & 2 & 1 & $0 \cdot 06$ & $0 \cdot 06$ & 32 & 8 & 4 & 4 & 2 & 4 & 4 & 1 & 1 & 81 \\
\hline PS 91-D305 & 1992 & $\mathrm{ND}$ & $\mathrm{ND}$ & $\mathrm{PF}$ & $\mathrm{m}$ & $\mathrm{ND}$ & 2 & 1 & $0 \cdot 06$ & $0 \cdot 06$ & 32 & 8 & 4 & 4 & 2 & 4 & 4 & 1 & 1 & 81 \\
\hline PS $184-D 306^{*}$ & 1992 & Munich & BA & CSF & $\mathrm{m}$ & $\mathrm{ND}$ & 1 & 1 & $0 \cdot 06$ & $0 \cdot 06$ & 16 & 8 & 4 & 4 & 2 & 4 & 4 & 1 & 1 & 81 \\
\hline PS 376 & 1992 & Berlin & B & BS & $\mathrm{m}$ & 65 & 1 & 1 & $0 \cdot 06$ & $0 \cdot 06$ & 32 & 4 & 4 & 4 & 2 & 4 & 4 & 1 & 1 & 81 \\
\hline PS 453 & 1992 & Hanover & LS & BS & $\mathrm{m}$ & 5 & 2 & 2 & $32 \dagger$ & 32 & 32 & 8 & 4 & 4 & 2 & 4 & 4 & 1 & 1 & 81 \\
\hline PS 570 & 1992 & Munich & BA & ND & $\mathrm{f}$ & 85 & 1 & 1 & 0.06 & $0 \cdot 06$ & 32 & 6 & 4 & 4 & 2 & 4 & 4 & 1 & 1 & 81 \\
\hline PS 853 & 1993 & Freiburg & BW & $\mathrm{ND}$ & $\mathrm{f}$ & 2 & 1 & 1 & 0.06 & $0 \cdot 06$ & 32 & 6 & 4 & 4 & 2 & 4 & 4 & 1 & 1 & 81 \\
\hline PS 999 & 1993 & Hanover & LS & Sputum & $\mathrm{m}$ & 66 & 1 & $0 \cdot 5$ & $0 \cdot 06$ & $0 \cdot 06$ & 32 & 8 & 4 & 4 & 2 & 4 & 4 & 1 & 1 & 81 \\
\hline PS 1005 & 1993 & Frankfurt: & $\mathrm{HE}$ & Blood & $\mathrm{ND}$ & $\mathrm{ND}$ & 1 & $0 \cdot 5$ & $0 \cdot 06$ & $0 \cdot 06$ & 32 & 32 & 4 & 4 & 2 & 4 & 4 & 1 & 1 & 81 \\
\hline PS 1058 & 1993 & Hanover & LS & BS & $\mathrm{m}$ & 66 & 1 & 1 & $0 \cdot 125$ & $0 \cdot 06$ & 32 & 16 & 4 & 4 & 2 & 4 & 4 & 1 & 1 & 81 \\
\hline PS 1271 & 1994 & Aachen & NRW & BS & $\mathrm{m}$ & 64 & 1 & 1 & $32 \dagger$ & 16 & $0 \cdot 5$ & 32 & 4 & 4 & 2 & 4 & 4 & 1 & 1 & 81 \\
\hline PS 1702 & 1995 & Aachen & NRW & Ear & $\mathrm{ND}$ & 6 & 2 & 1 & $32 \S$ & $0 \cdot 25$ & 8 & 4 & 4 & 4 & 2 & 4 & 4 & 1 & 1 & 81 \\
\hline PS 1762 & 1995 & Hanover & LS & Blood & $\mathrm{m}$ & 50 & 2 & 1 & $0 \cdot 06$ & $0 \cdot 06$ & 4 & 4 & 4 & 4 & 2 & 4 & 4 & 1 & 1 & 81 \\
\hline PS 1923 & 1995 & Bonn & NRW & ND & $\mathrm{ND}$ & $\mathrm{ND}$ & 1 & 1 & $0 \cdot 06$ & $0 \cdot 06$ & 8 & 4 & 4 & 4 & 2 & 4 & 4 & 1 & 1 & 81 \\
\hline PS 2352 & 1996 & Bonn & NRW & Sputum & $\mathrm{m}$ & 59 & 1 & 1 & $0 \cdot 06$ & $0 \cdot 06$ & 4 & 4 & 4 & 4 & 2 & 4 & 4 & 1 & 1 & 81 \\
\hline PS 2361 & 1996 & Hanover & LS & Nasop. & $\mathrm{m}$ & 4 & 1 & 1 & $32 \dagger$ & 32 & 16 & 4 & 4 & 4 & 2 & 4 & 4 & 1 & 1 & 81 \\
\hline PN 958 & 1997 & Dillingen & BA & BS & $\mathrm{f}$ & 72 & 1 & 1 & $0 \cdot 06$ & $0 \cdot 06$ & 32 & 4 & 4 & 4 & 2 & 4 & 4 & 1 & 1 & 81 \\
\hline RESP 203 & 1998 & ND & $\mathrm{ND}$ & Nasop. & $\mathrm{m}$ & 2 & 2 & $0 \cdot 25$ & $32 \dagger$ & 32 & 16 & 8 & 4 & 4 & 2 & 4 & 4 & 1 & 1 & 81 \\
\hline RKI 277 & 1998 & Stuttgart & BW & Blood & $\mathrm{m}$ & 2 & $0 \cdot 5$ & $0 \cdot 5$ & $2 \S$ & $0 \cdot 06$ & $0 \cdot 25$ & 4 & 4 & 4 & 2 & 4 & 4 & 1 & 1 & 81 \\
\hline RESP 950 & 1999 & Weiden & BA & Sinus & $\mathrm{m}$ & 55 & 2 & $0 \cdot 25$ & $0 \cdot 06$ & $0 \cdot 06$ & 32 & 4 & 4 & 4 & 2 & 4 & 4 & 1 & 1 & 81 \\
\hline \multicolumn{21}{|l|}{ ST 277} \\
\hline PS $335-D 310$ & 1992 & Aachen & NRW & Conj. & $\mathrm{m}$ & 1 & $0 \cdot 25$ & 0.06 & $0 \cdot 06$ & $0 \cdot 06$ & $0 \cdot 125$ & 2 & 7 & 13 & 8 & 6 & 6 & 12 & 8 & 277 \\
\hline PS 2188 & 1996 & Aachen & NRW & Blood & $\mathrm{m}$ & $\mathrm{ND}$ & $0 \cdot 25$ & $0 \cdot 06$ & $0 \cdot 06$ & $0 \cdot 06$ & $0 \cdot 5$ & 1 & 7 & 13 & 8 & 6 & 6 & 12 & 8 & 277 \\
\hline PS 2211 & 1996 & Münster & NRW & Blood & $\mathrm{m}$ & 53 & $0 \cdot 25$ & $0 \cdot 25$ & $0 \cdot 06$ & $0 \cdot 06$ & $0 \cdot 5$ & $0 \cdot 125$ & 7 & 13 & 8 & 6 & 6 & 12 & 8 & 277 \\
\hline PS 2216 & 1996 & Cologne & NRW & Wound & $\mathrm{m}$ & 35 & $0 \cdot 25$ & 0.06 & 0.06 & $0 \cdot 06$ & $0 \cdot 5$ & $0 \cdot 25$ & 7 & 13 & 8 & 6 & 6 & 12 & 8 & 277 \\
\hline
\end{tabular}




\begin{tabular}{|c|c|c|c|c|c|c|c|c|c|c|c|c|c|c|c|c|c|c|c|c|}
\hline PN 1003 & 1997 & Erlangen & BA & Blood & ND & $\mathrm{ND}$ & $0 \cdot 25$ & 0.06 & 0.06 & 0.06 & $0 \cdot 125$ & 2 & 7 & 13 & 8 & 6 & 6 & 12 & 8 & 277 \\
\hline PN 962 & 1997 & Erlangen & BA & Conj. & ND & $1 \mathrm{~m}$ & $0 \cdot 25$ & 0.06 & $0 \cdot 06$ & $0 \cdot 06$ & $0 \cdot 5$ & 4 & 7 & 13 & 8 & 6 & 6 & 12 & 8 & 277 \\
\hline RESP 4 & 1998 & Hamburg & $\mathrm{HH}$ & Nasop. & $\mathrm{f}$ & 5 & $0 \cdot 25$ & $0 \cdot 125$ & $0 \cdot 125$ & $0 \cdot 125$ & $0 \cdot 25$ & 1 & 7 & 13 & 8 & 6 & 6 & 12 & 8 & 277 \\
\hline RKI 629 & 2000 & Heidelberg & BW & Blood & $\mathrm{f}$ & 5 & $0 \cdot 25$ & 0.06 & $0 \cdot 125$ & $0 \cdot 06$ & $0 \cdot 5$ & 8 & 7 & 13 & 8 & 6 & 6 & 12 & 8 & 277 \\
\hline \multicolumn{21}{|l|}{ ST 242} \\
\hline PS 1953 & 1995 & Aachen & NRW & $\mathrm{ND}$ & ND & $\mathrm{ND}$ & 1 & 1 & $32 \dagger$ & 32 & 32 & 1 & 15 & 29 & 4 & 21 & 30 & 1 & 14 & 242 \\
\hline PS 2006 & 1995 & Frankfurt: & $\mathrm{HE}$ & $\mathrm{ND}$ & ND & $\mathrm{ND}$ & $0 \cdot 25$ & $0 \cdot 25$ & $32 \dagger$ & 32 & 32 & $0 \cdot 5$ & 15 & 29 & 4 & 21 & 30 & 1 & 14 & 242 \\
\hline \multicolumn{21}{|l|}{ ST 142} \\
\hline RKI 530 & 1999 & Dortmund & NRW & Blood & $\mathrm{m}$ & 1 & $0 \cdot 25$ & $0 \cdot 125$ & $0 \cdot 125$ & 0.06 & 8 & $0 \cdot 125$ & 7 & 5 & 8 & 6 & 6 & 12 & 8 & $142 \|$ \\
\hline RKI 531 & 1999 & Dortmund & NRW & Blood & $\mathrm{m}$ & $1 \mathrm{~m}$ & $0 \cdot 25$ & $0 \cdot 06$ & 0.06 & $0 \cdot 06$ & 8 & $0 \cdot 125$ & 7 & 5 & 8 & 6 & 6 & 12 & 8 & $142 \|$ \\
\hline \multicolumn{21}{|l|}{ ST 323} \\
\hline PS 943 & 1993 & Hanover & LS & Sputum & $\mathrm{m}$ & 34 & $0 \cdot 125$ & $0 \cdot 125$ & 0.06 & $0 \cdot 06$ & 4 & 2 & 2 & 13 & 8 & 12 & 6 & 6 & 18 & 323 \\
\hline \multicolumn{21}{|l|}{ New STs } \\
\hline PS 1374 & 1994 & Jena & TU & ND & $\mathrm{m}$ & 5 & $0 \cdot 5$ & $0 \cdot 5$ & $0 \cdot 06$ & $0 \cdot 06$ & $0 \cdot 5$ & $0 \cdot 5$ & 1 & 5 & 9 & 5 & 6 & 3 & 65 & 353 \\
\hline PS 2031 & 1996 & Frankfurt: & $\mathrm{HE}$ & CSF & $\mathrm{m}$ & 9 & $0 \cdot 25$ & $0 \cdot 125$ & 0.06 & $0 \cdot 06$ & $0 \cdot 5$ & 4 & 1 & 5 & 9 & 5 & 15 & 3 & 8 & 354 \\
\hline RESP 798 & 1999 & Weiden & $\mathrm{BA}$ & Nasop. & $\mathrm{m}$ & 4 & $0 \cdot 125$ & $0 \cdot 06$ & 0.06 & 0.06 & $0 \cdot 25$ & $0 \cdot 125$ & 1 & 8 & 4 & 2 & 6 & 4 & 6 & 355 \\
\hline RESP 801 & 1999 & Neukirchen & $\mathrm{ND}$ & Eye & $\mathrm{m}$ & 1 & $0 \cdot 125$ & $0 \cdot 06$ & 0.06 & $0 \cdot 125$ & $0 \cdot 125$ & 1 & 1 & 13 & 27 & 20 & 6 & 1 & 1 & 356 \\
\hline RKI 243 & 1998 & Stuttgart & BW & Blood & $\mathrm{ND}$ & 1 & $0 \cdot 25$ & $0 \cdot 125$ & 0.06 & 0.06 & $0 \cdot 06$ & $0 \cdot 125$ & 7 & 13 & 8 & 6 & 15 & 20 & 14 & 357 \\
\hline PS 2260 & 1996 & Erlangen & $\mathrm{BA}$ & Conj. & $\mathrm{m}$ & $4 \mathrm{~m}$ & $0 \cdot 25$ & $0 \cdot 25$ & 0.06 & $0 \cdot 06$ & $0 \cdot 5$ & $0 \cdot 125$ & 7 & 13 & 8 & 6 & 15 & $74^{* *}$ & 6 & 358 \\
\hline RKI 425 & 1999 & Glauchau & SA & Blood & $\mathrm{m}$ & 3 & $0 \cdot 25$ & $0 \cdot 06$ & 0.06 & $0 \cdot 06$ & $0 \cdot 5$ & $0 \cdot 125$ & 7 & 13 & 8 & 6 & 6 & 20 & 8 & $359 \|$ \\
\hline RKI 682 & 1999 & Munich & $\mathrm{BA}$ & Blood & $\mathrm{m}$ & 2 & $0 \cdot 25$ & $0 \cdot 06$ & $0 \cdot 125$ & $0 \cdot 06$ & $0 \cdot 5$ & $0 \cdot 125$ & 7 & 13 & 8 & 6 & 6 & $65^{* *}$ & 8 & $360 \|$ \\
\hline PS 275-D307 & 1992 & Erlangen & $\mathrm{BA}$ & CSF & $\mathrm{f}$ & 70 & $0 \cdot 25$ & $0 \cdot 06$ & 0.06 & $0 \cdot 06$ & 2 & 3 & 7 & 13 & 8 & 6 & 6 & 6 & 8 & $361 \|$ \\
\hline PS 278-D308 & 1992 & Berlin & B & Wound & $\mathrm{m}$ & 86 & $0 \cdot 25$ & $0 \cdot 25$ & $0 \cdot 06$ & $0 \cdot 06$ & 2 & 8 & 7 & 13 & 8 & 6 & 6 & 6 & 8 & $361 \|$ \\
\hline RESP 95 & 1998 & Leverkusen & NRW & Nasop. & $\mathrm{f}$ & 32 & $0 \cdot 125$ & $0 \cdot 125$ & $8 \S$ & $0 \cdot 1253$ & & 1 & 15 & 13 & 4 & 21 & 30 & 1 & 14 & $362 \dagger \dagger$ \\
\hline
\end{tabular}

${ }^{*}$ Identical to strain D306-W-23F in MLST database.

$\dagger$ †rm-positive.

†Frankfurt am Main.

§mef-positive.

॥SLV of ST 277.

- SLV of ST 37 and ST 33.

**New allele seen to date only in Germany.

$\dagger \dagger$ SLV of ST 242. 
by MLST (or, in some cases, differed at a single locus) from multiply resistant strains from Taiwan or the USA. Only five strains were not closely related to any in the MLST database.

Data on the epidemiology of pneumococcal disease in Germany are scant to date. Reichmann et al. (1995) compared penicillin-resistant $S$. pneumoniae strains isolated in different parts of Germany between 1982 and 1992 with penicillin-resistant isolates, mainly of serogroups 6, 9, 14, 19 and 23, from other European countries. Strains were characterized by their serotypes, antibiotic resistance patterns, penicillin-binding protein properties and multilocus enzyme electrophoresis (MLEE). Eleven of the 14 penicillinresistant German isolates were assigned to five genotypes that were also isolated in other countries. The study by Reichmann et al. (1995) included five serotype 23F strains of $S$. pneumoniae, which are included in the collection of strains in this investigation. Serotype 23F strains PS 91-D305 and PS 184-D306, classified as MLEE type 2, were shown by MLST to be equivalent to ST 81 (the Spain $^{23} \mathrm{~F}-1$ clone). MLEE types 21 and 22 were demonstrated by MLST to be closely related to and to correspond to ST 277 (strain PS 335D310) and an SLV of ST 277 (ST 361, strains PS 275-D307 and PS 278-D308), respectively. These observations underscore the high discriminative power of both MLEE and MLST and document that both methods lead to identical results when investigating clonal relatedness of pneumococcal isolates.

MLST was used by Zhou et al. (2000) to characterize isolates of the major Spanish clones of penicillin-resistant and multiply antibiotic-resistant $S$. pneumoniae strains, including previously characterized isolates of the Spanish ${ }^{23 \mathrm{~F}}-1$ clone (Coffey et al., 1996). Fifteen out of 16 of these isolates had an identical allelic profile (ST 81, profile 4-4-2-4-4-1-1), which was considered to be the typical allelic profile of the Spanish ${ }^{23 \mathrm{~F}}-1$ clone.

Data from this study clearly document that most penicillin non-susceptible strains are closely related to those found in Spain. Results of our study also underscore that travelling may contribute to the spread of resistance. Germans spent 82.6 billion DM (38.4 billion US\$) on travel in 1998. For many years, the main holiday destination for the German population has been Spain; a total of 5.7 million Germans flew to Spain (19.7\% of all destinations) in 1998. It is therefore likely that resistant strains are continuously imported into Germany, presumably mainly from Spain. Nevertheless, it remains unclear why these strains do not spread within Germany to a greater extent.

Differing antibiotic consumption in European countries may largely account for these differences. Our group studied the relationship between penicillin and erythromycin resistance among isolates of $S$. pneumoniae, relative to macrolide consumption, in Germany over 9 years (1992-2000) (Granizo et al., 2000; Reinert et al., 2002). A significant relationship was observed between erythromycin resistance and macrolide consumption. Although that ecological analysis was not able to demonstrate a causal relationship between antibiotic consumption and development of resistance, it suggested that overuse of certain specific antibiotics is more likely to be related to the increase in resistance. High prevalence of the Spanish 23F-clone in Germany may also be due to the relatively high consumption of tetracycline, which was widely used in Germany in the mid-1990s, as this $23 \mathrm{~F}$ clone is also tetracycline-resistant. A study by Cars et al. (2001) has demonstrated that sales of antibiotics varied more than fourfold within Europe; France and Spain had the highest sales, whereas the Netherlands, Denmark, Sweden and Germany had the lowest (Cars et al., 2001). In addition, the relatively high frequency of pre-school children staying at home and not at day-care centres may contribute to the lower level of antibiotic resistance in Germany.

In conclusion, penicillin non-susceptible serotype 23F isolates in Germany can be assigned to two major clones, but other strains also contribute significantly to resistance. Some isolates appear to have not yet been identified outside Germany. The findings of the present study underscore that, despite the low level of $\beta$-lactam resistance, penicillinresistant serotype 23F ST81, which is responsible for high resistance rates reported from other European countries, is present in Germany. Consequently, to preserve the currently low antimicrobial resistance levels among clinical isolates of S. pneumoniae, prudent usage of antimicrobials in Germany is warranted.

\section{ACKNOWLEDGEMENTS}

Presented in part at the 11th European Congress of Clinical Microbiology and Infectious Diseases, Istanbul, Turkey, 1-4 April 2001. The authors thank Nelli Neuberger, Maria Lemperle and Claudia Cremer for excellent technical assistance and Susan Griesbach, Münster, for copy editing. The study was supported by grant RKI-415/1369235 from the German Minister of Health (Bundesminister für Gesundheit) and by a CAP-net grant [German Ministry of Education and Research (Bundesministerium für Bildung und Forschung)].

\section{REFERENCES}

Andrews, J., Ashby, J., Jevons, G., Lines, N. \& Wise, R. (1999). Antimicrobial resistance in gram-positive pathogens isolated in the UK between October 1996 and January 1997. J Antimicrob Chemother 43, 689-698.

Cars, O., Molstad, S. \& Melander, A. (2001). Variation in antibiotic use in the European Union. Lancet 357, 1851-1853.

Coffey, T. J., Berrón, S., Daniels, M., Garcia-Leoni, M. E., Cercenado, E., Bouza, E., Fenoll, A. \& Spratt, B. G. (1996). Multiply antibiotic-resistant Streptococcus pneumoniae recovered from Spanish hospitals (19881994): novel major clones of serotypes 14, 19F and 15F. Microbiology $142,2747-2757$

Enright, M. C. \& Spratt, B. G. (1998). A multilocus sequence typing scheme for Streptococcus pneumoniae: identification of clones associated with serious invasive disease. Microbiology 144, 3049-3060.

Granizo, J. J., Aguilar, L., Casal, J., Dal-Ré, R. \& Baquero, F. (2000). Streptococcus pyogenes resistance to erythromycin in relation to macrolide consumption in Spain (1986-1997). J Antimicrob Chemother 46, 959-964. 
Henriques, B., Kalin, M., Örtqvist, А̊. \& 8 other authors (2000). Molecular epidemiology of Streptococcus pneumoniae causing invasive disease in 5 countries. J Infect Dis 182, 833-839.

Hermans, P. W. M., Sluijter, M., Elzenaar, K. \& 8 other authors (1997). Penicillin-resistant Streptococcus pneumoniae in the Netherlands: results of a 1-year molecular epidemiologic survey. J Infect Dis 175, 1413-1422.

Johnson, A. P. (1998). Antibiotic resistance among clinically important gram-positive bacteria in the UK. J Hosp Infect 40, 17-26.

Klugman, K., Goldstein, F., Kohno, S. \& Baquero, F. (1997). The role of fourth-generation cephalosporins in the treatment of infections caused by penicillin-resistant streptococci. Clin Microbiol Infect 3 (Suppl. 1), S48-S60.

Maiden, M. C. J., Bygraves, J. A., Feil, E. \& 10 other authors (1998). Multilocus sequence typing: a portable approach to the identification of clones within populations of pathogenic microorganisms. Proc Natl Acad Sci U S A 95, 3140-3145.

McGee, L., McDougal, L., Zhou, J. \& 8 other authors (2001). Nomenclature of major antimicrobial-resistant clones of Streptococcus pneumoniae defined by the pneumococcal molecular epidemiology network. J Clin Microbiol 39, 2565-2571.

NCCLS (2000). Methods for Dilution Antimicrobial Susceptibility Tests for Bacteria that Grow Aerobically (M7-A4); Approved Standard (M100S8), 4th edn. Wayne, PA: NCCLS.

Neufeld, F. (1902). Über die Agglutination der Pneumokokken und über die Theorie der Agglutination. Z Hyg Infektionskr 34, 54-72 (in German).

Nielsen, S. V. \& Henrichsen, J. (1992). Capsular types of Streptococcus pneumoniae isolated from blood and CSF during 1982-1987. Clin Infect Dis 15, 794-798.

Pradier, C., Dunais, B., Carsenti-Etesse, H. \& Dellamonica, P. (1997). Pneumococcal resistance patterns in Europe. Eur J Clin Microbiol Infect Dis 16, 644-647.

Provvedi, R., Manganelli, R. \& Pozzi, G. (1996). Characterization of conjugative transposon Tn5251 of Streptococcus pneumoniae. FEMS Microbiol Lett 135, 231-236.

Reichmann, P., Varon, E., Günther, E., Reinert, R. R., Lütticken, R., Marton, A., Geslin, P., Wagner, J. \& Hakenbeck, R. (1995). Penicillinresistant Streptococcus pneumoniae in Germany: genetic relationship to clones from other European countries. J Med Microbiol 43, 377-385.
Reinert, R. R., Kaufhold, A. \& Kierdorf, H. (1992). Penicillin-resistant pneumococcus in community-acquired bacteremic pneumonia in Germany. Infection 20, 238-239.

Reinert, R. R., Queck, A., Kaufhold, A., Kresken, M. \& Lütticken, R. (1995). Antimicrobial resistance and type distribution of Streptococcus pneumoniae isolates causing systemic infections in Germany, 19921994. Clin Infect Dis 21, 1398-1401.

Reinert, R. R., Simic, S., Al-Lahham, A., Reinert, S., Lemperle, M. \& Lütticken, R. (2001). Antimicrobial resistance of Streptococcus pneumoniae recovered from outpatients with respiratory tract infections in Germany from 1998 to 1999: results of a national surveillance study. J Clin Microbiol 39, 1187-1189.

Reinert, R. R., Al-Lahham, A., Lemperle, M., Tenholte, C., Briefs, C., Haupts, S., Gerards, H. H. \& Lütticken, R. (2002). Emergence of macrolide and penicillin resistance among invasive pneumococcal isolates in Germany. J Antimicrob Chemother 49, 61-68.

Shi, Z.-Y., Enright, M. C., Wilkinson, P., Griffiths, D. \& Spratt, B. G. (1998). Identification of three major clones of multiply antibioticresistant Streptococcus pneumoniae in Taiwanese hospitals by multilocus sequence typing. J Clin Microbiol 36, 3514-3519.

Tait-Kamradt, A., Clancy, J., Cronan, M., Dib-Hajj, F., Wondrack, L., Yuan, W. \& Sutcliffe, J. (1997). mefE is necessary for the erythromycinresistant M phenotype in Streptococcus pneumoniae. Antimicrob Agents Chemother 41, 2251-2255.

Trieu-Cuot, P., Poyart-Salmeron, C., Carlier, C. \& Courvalin, P. (1990). Nucleotide sequence of the erythromycin resistance gene of the conjugative transposon Tn1545. Nucleic Acids Res 18, 3660.

von Kries, R., Siedler, A., Schmitt, H. J. \& Reinert, R. R. (2000). Proportion of invasive pneumococcal infections in German children preventable by pneumococcal conjugate vaccines. Clin Infect Dis 31, 482-487.

Widdowson, C. A. \& Klugman, K. P. (1998). The molecular mechanisms of tetracycline resistance in the pneumococcus. Microb Drug Resist 4, 79-84.

Widdowson, C. A., Klugman, K. P. \& Hanslo, D. (1996). Identification of the tetracycline resistance gene, tet $(\mathrm{O})$, in Streptococcus pneumoniae. Antimicrob Agents Chemother 40, 2891-2893.

Zhou, J., Enright, M. C. \& Spratt, B. G. (2000). Identification of the major Spanish clones of penicillin-resistant pneumococci via the Internet using multilocus sequence typing. J Clin Microbiol 38, 977-986. 